\title{
Statistical Image Reconstruction in PET with Compensation for Missing Data
}

\author{
PE Kinahan ${ }^{1}$ Member, IEEE, JA Fessler ${ }^{2}$ Member, IEEE, and JS Karp ${ }^{3}$ Member, IEEE \\ Universities of ${ }^{1}$ Pittsburgh, ${ }^{2}$ Michigan, and ${ }^{3}$ Pennsylvania
}

\begin{abstract}
We present the results of combining volume imaging with the PENN-PET scanner with statistical image reconstruction methods such as the penalized weighted least squares (PWLS) method. The goal of this particular combination is to improve both classification and estimation tasks in PET imaging protocols where image quality is dominated by spatially. variant system responses and/or measurement statistics. The PENN-PET scanner has strongly spatially-varying system behavior due to its volume imaging design and the presence of detector gaps. Statistical methods are easily adapted to this scanner geometry, including the detector gaps, and have also been shown to have improved bias/variance trade-offs compared to the standard filtered-backprojection (FBP) reconstruction method. The PWLS method requires fewer iterations and may be more tolerant of errors in the system model than other statistical methods. We present results demonstrating the improvement in image quality for PWLS image reconstructions of data from the PENN-PET scanner.
\end{abstract}

\section{INTRODUCTION}

\section{A. Statistical Image Reconstruction Methods.}

It is well recognized that statistical reconstruction algorithms, such as maximum-likelihood via expectation maximization (ML-EM), are particularly helpful in cases where measurement statistics dominate image quality [1]. To realize their full potential, these algorithms depend on accurate system models that describe the system response function and expected measurement statistics for a known object.

Statistical iterative methods assume that the expected value of the projection data is given by $\overline{\mathbf{y}}=\mathrm{E}[\mathbf{y}]=\mathbf{A x}$, where $\mathbf{x}=\left\{x_{i} \mid i=1, \ldots, n\right\}$ is a vector of the $n$ voxel values of the image, $\mathbf{y}=\left\{y_{j} \mid j=1, \ldots, m\right\}$ are the $m$ projection values, and $\mathbf{A}=\left\{A_{j i}\right\}$ is the $m \times n$ system matrix that gives the probability of a photon emitted from voxel $i$ being detected in projection bin $j$. The measured projection data, on the other hand, is a random vector drawn from the probability distribution function (PDF) given by an assumed statistical model. The ML-EM method assumes that the system model, A, accurately relates the PDF of the estimated image data to the Poisson-distributed sinogram data and seeks to maximize the log likelihood functional given by:

$$
\Phi(\mathbf{x})=\sum_{j} y_{j} \ln \left(\sum_{i} A_{j i} x_{i}\right)-\sum_{i} A_{j i} x_{i},
$$

\footnotetext{
${ }^{1}$ Department of Radiology, 200 Lothrop Street, Pittsburgh, PA, 15213. This work was supported by a grant from the Whitaker Foundation, NIH grant CA-60711, and DOE grant DE-FG0288ER60642.
}

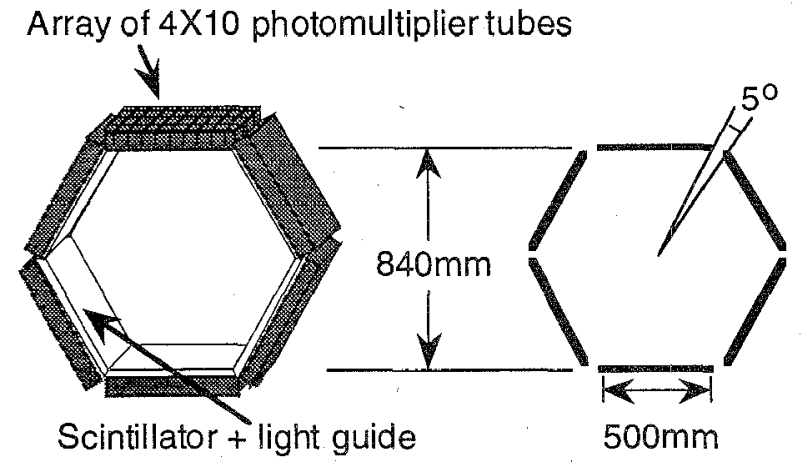

Fig. 1. Geometry of the PENN-PET scanner.

possibly with the inclusion of an a priori regularizing term included via a maximum a posteriori (MAP) formulation of the estimation problem.

The expected measurement statistics are usually assumed to be Poisson, often with the inclusion of attenuation and other effects, and the system response function of PET scanners is usually assumed to spatially invariant. Neither of these assumptions are completely accurate in practice. One solution is to derive a more accurate system model that explicitly takes into account all effects such as attenuation, scattered and random coincidences, resolution, and others [2].

An alternative approach that may be may be more tolerant of inaccuracies in system models that do not properly account for all effects is to assume Gaussian statistics and minimize a penalized weighted least squares (PWLS) objective function, rather than a Poisson likelihood objective function [3]. The PWLS objective function, essentially a regularized $\chi^{2}$ functional, is given by,

$$
\Phi(\mathbf{x})=-\sum_{j}\left(\sum_{i} A_{j i} x_{i}-y_{j}\right)^{2} \sigma_{j}^{-2}-\beta U(\mathbf{x}),
$$

where $\sigma=\left\{\sigma_{j} \mid j=1, \ldots, m\right\}$ are the standard deviations for the projection data and $\beta$ controls the influence of the modified quadratic roughness penalty functional, $U(\mathbf{x})$, described by Fessler and Rogers [4]. The standard deviations are estimated based on the corrections applied to the raw sinogram data during the processing steps prior to the image reconstruction, and the effect of weighting the differences between estimated and measured projections takes into account the statistical quality of the data.

Minimization of a PWLS objective function by successive over-relaxation (SOR) has been shown to be effective in cases where the data statistics are not Poisson [3]. The SOR algorithm is a coordinate-descent method where image pixels are estimated in place. In other words, $\Phi\left(x_{i}\right)$ is minimized independently for each image pixel, $i$, in turn. This has the advantages of estimating the image data directly, and allowing for the straightforward application of a non-negativity constraint on the estimated image. 


\section{B. The PENN-PET Scanner System Model}

The PENN-PET scanner at the University of Pennsylvania is a volume-imaging PET camera with an axial field of view (FOV) of $12.8 \mathrm{~cm}$, a transverse FOV of $50 \mathrm{~cm}$ and gaps in coverage of $\sim 5$ deg where the 6 planar detectors meet as illustrated in fig. 1 [5].

Since the scanner has no septa, data are acquired in 3D mode, with on-line rebinning of the $3 \mathrm{D}$ data into standard $2 \mathrm{D}$ sinograms via the single-slice rebinning (SSRB) algorithm [6]. The gaps in the detector coverage lead to the missing sinogram regions shown in fig. 2 .

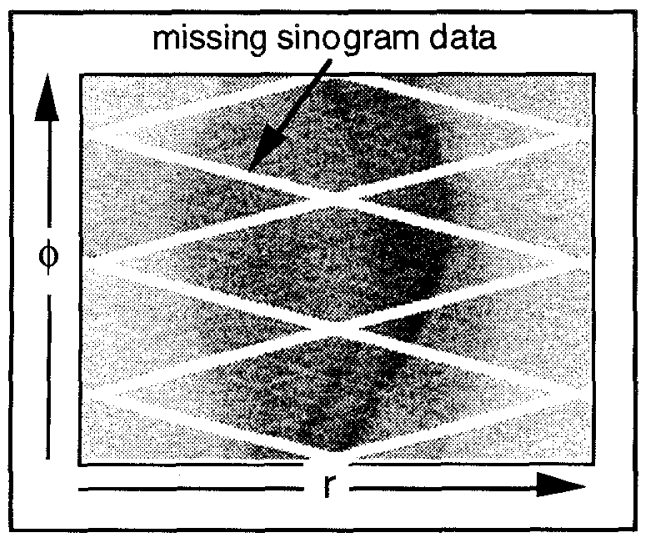

Fig. 2 Sinogram of a cardiac study showing the missing data due to 5 deg detector gaps.

The corrected sinogram data, $\mathbf{y}_{c}$, can be described by:

$$
\mathbf{y}_{c}=\mathbf{a} \cdot\left(\mathbf{e}_{1} \cdot \mathbf{e}_{2} \cdot \mathbf{e}_{3} \cdot \mathbf{y}_{S S R B}-\mathbf{b}\right)
$$

where $\mathbf{y}_{S S R B}$ is the SSRB rebinned sinogram data, $\mathbf{b}$ is the fitted scatter and randoms subtraction [5], $\mathbf{a}$ is the attenuation correction, and $\mathbf{e}_{i}$ are efficiency corrections for $\mathrm{i}=1$ : axial variation due to SSRB rebinning of volume acquisition, $i=2$ : sampling pattern from on-line rebinning, and $i=3$ : detector efficiency variations.

For conventional filtered-backprojection (FBP) image reconstruction, the constrained Fourier space method (CFSM), based on consistency conditions in the Fourier transform of the sinogram data, is used to estimate the missing sinogram data $[5,7]$.

Statistical reconstruction methods are easily adapted to missing data situations, especially if there are still more measured sinogram samples than image voxels, and are particularly advantageous in protocols with poor statistics, such as whole-body imaging. These methods can directly incorporate constraints and prior information to partially compensate for the missing rays, unlike FBP, which must start with a complete data sinogram. The PWLS+SOR method is particularly well suited to reconstruct data from the PENN-PET scanner, as the weights associated with the regions of missing sinogram data can simply be set to zero. The effect of the correction steps in equation (2) on the nonzero weights in equation (1) are straightforward to calculate and are described by Fessler [3]. To account for the effect of attenuation correction, for example, we pre-corrected the sinogram data $\mathbf{y}_{c}$, for attenuation and adjusted the weights $\boldsymbol{\sigma}^{-2}=\left\{\sigma_{j}^{-2} \mid j=1, \ldots, m\right\}$, appropriately.

In addition, the PWLS+SOR may be more tolerant than other statistical methods of inaccuracies in the system model introduced by the SSRB rebinning and subsequent renormalization steps, since it only takes into account the mean and variance of the sinogram data, and does not expect the entire probability distribution function (e.g. a Poisson distribution) to be characterized.

\section{ESTIMATION OF MISSING DATA}

To compare the effectiveness of the PWLS+SOR and CFSM-FBP methods in reconstructing the incomplete data from the PENN-PET scanner, images were reconstructed of a simulated cylinder. (The notation PWLS "+" SOR symbolizes the addition of a positivity constraint in the SOR algorithm). The simulation did not include any other effects than Poisson noise and detector gaps. The reduction in statistical noise is shown in figs. 3 and 4 . The missing sinogram data are effectively compensated for with both methods, as there is sufficient frequency information to for the CFSM algorithm to estimate the missing sinogram data [7].

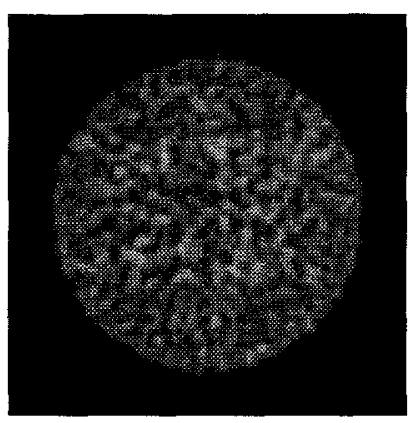

Fig. 3a. Simulated uniform cylinder data reconstructed by CFSM-FBP method.

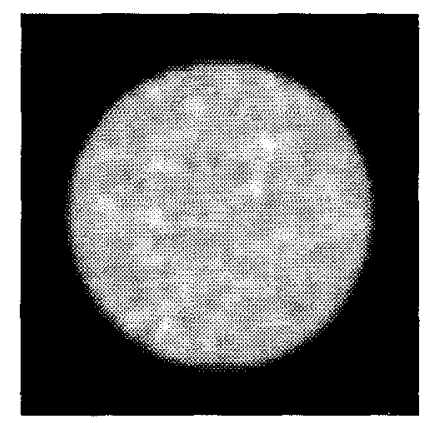

Fig. 3b. Simulated uniform cylinder data reconstructed by the PWLS+SOR method.

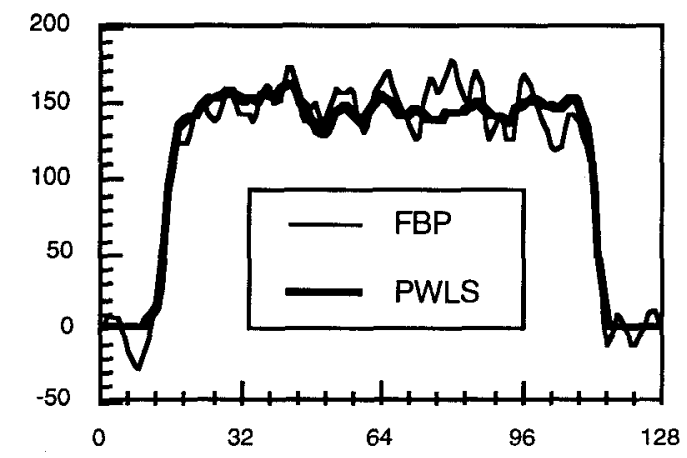

Fig. 4. Transverse line profiles through the center of the images (shown above) reconstructed by FBP and PWLS. Pixels are $2 \mathrm{~mm}$.

Figs. 3 and 4 also illustrate a reduction in the image noise for the PWLS+SOR method for similar image resolutions as the CFSM-FBP image, where the matched resolution is shown by the closeness of the profiles at the edges of the cylinder. 


\section{POINT SPREAD FUNCTION}

Reconstructing an image of a point source with missing data is more challenging than for a cylinder, as there are more missing spatial frequencies in the sinogram data. To investigate the effects on the PSF, a slightly off-center line was imaged in air and reconstructed without applying any corrections for scatter or attenuation. The reconstructed images were carefully matched between the two methods to have the same FWHM $(12 \mathrm{~mm})$ and FWTM $(25 \mathrm{~mm})$, which are typical of clinical whole-body imaging, and is the same resolution used in the phantom and patient images below. Surface plots of the central plane of a 3D line source image reconstructed by both methods are shown in fig. 5 .

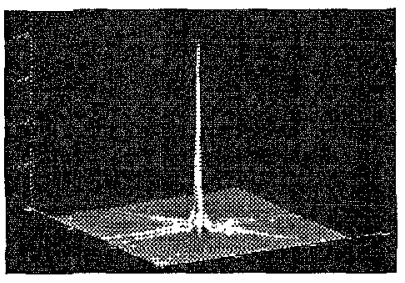

CFSM-FBP

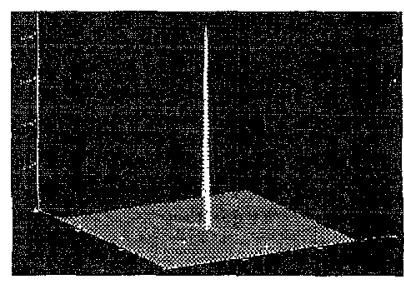

PWLS+SOR
Fig 5. Surface plot of central plane of a 3D line source image reconstructed by both methods with equal resolution as determined by FWHM and FWTM. The plots are displayed on the same scale.

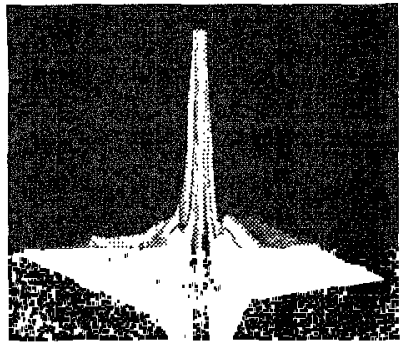

CFSM-FBP

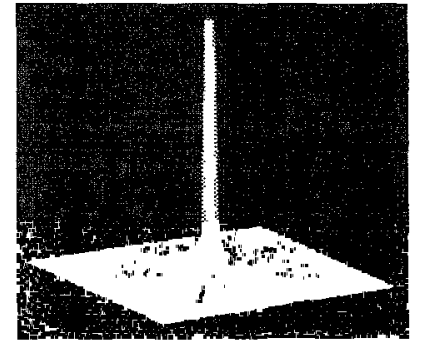

PWLS+SOR
Fig 6. Same plots as shown in fig. 5, but thresholded to the FWTM for each image. The plots are displayed on the same scale.

The increased fluctuations in the tails for the CFSM-FBP algorithm are evident in fig. 6 , which shows the same plots as in fig. 5 but thresholded to the FWTM for each image. The differences between the two methods are due to the nonnegativity constraint, which in principle could also be applied to the CFSM-FBP method using the technique developed by O'Sullivan et al [8].

\section{RESOLUTION, CONTRAST, AND NOISE}

To evaluate the effects of the non-negativity constraint and the incorporation of statistical noise properties by the PWLS+SOR method, a cylinder with a hot line source (offcenter), a warm background and a cold cylindrical insert was imaged on the PENN-PET scanner to simultaneously measure resolution, contrast, and noise. The phantom was scanned for two different imaging conditions, one of low statistics $(\sim 50 \mathrm{k}$ events per image slice), and one or moderate statistics ( $250 \mathrm{k}$ events per image slice). The images were reconstructed over a range of parameters to control the usual bias/resolution tradeoff. For the CFSM-FBP method this was done by setting the cut-off frequency of the apodizing Hamming window to 2.0,
$1.0,0.67,0.5$, and 0.4 of the Nyquist frequency $\left(0.25 \mathrm{~mm}^{-1}\right)$, while for the PWLS+SOR method this was done by controlling the influence of the regularizing term by setting the parameter $\beta$ in equation (1) to $2^{-q}$, where $q=14.0,12.8$, $9.0,7.0,5.9$, and 4.7. For the SOR minimization algorithm, 20 iterations were used, although 10 iterations would likely have given similar results.

The PSF of a PET scanner (with or without detector gaps) is not well characterized by estimating a FWHM from a fitted Gaussian profile. This is especially true for asymmetric PSFs of off-center points. For our purposes resolution was calculated using

$$
\mathrm{FWHM}=\sqrt{\left(\mathrm{FWHM}_{\mathrm{R}}^{2}+\mathrm{FWHM}_{\mathrm{T}}^{2}\right) / 2}
$$

where $\mathrm{FWHM}_{\mathrm{R}}$ and $\mathrm{FWHM}_{\mathrm{T}}$ are the radial and tangential FWHM directly measured using linear interpolation on profiles through the reconstructed line source. This rootmean-square measure tends to penalize more asymmetricallyshaped PSFs than a simple average of the radial and tangential FWHM values. A similar measure was used to determine the FWTM.

Contrast was measured from ROIs of the same diameter as the cold cylindrical insert placed over the background and the cold insert (determined from a high statistics scan), and calculated as the ratio (background - cold)/background.

Noise was calculated as the standard deviation of the background or cold ROIs normalized to the background level. The validity of this type of measurement is discussed below.

\section{A. Resolution versus Noise}

Fig. 7 shows no significant difference in resolution (FWHM and FWTM as calculated by equation 3) versus noise between the CFSM-FBP and PWLS+SOR methods. For the CFSM-FBP algorithm the trade-off was controlled by changing the cut-off frequency of the apodizing Hamming window, while for the PWLS+SOR method this was done by changing the parameter $\beta$ in equation (1) as described above. Also shown in fig. 7 are the FWHM and FWTM corresponding to the reconstruction parameters used in section $\mathrm{V}$, as indicated by the vertical arrows.

\section{B. Contrast versus Noise}

Fig. 8 shows the contrast vs. noise for the background and cold regions for the low and moderate statistics cases. The PWLS+SOR demonstrates an improved contrast/noise ratio. Similar to fig. 7, vertical arrows for the moderate statistics case indicate the contrast corresponding to the reconstruction parameters that are used in section $\mathrm{V}$.

\section{Contrast versus Resolution}

Fig. 9 directly compares the contrast versus resolution (FWHM as calculated by equation 3 ) for the two methods, and shows an improved contrast for the PWLS+SOR method for a given resolution. 


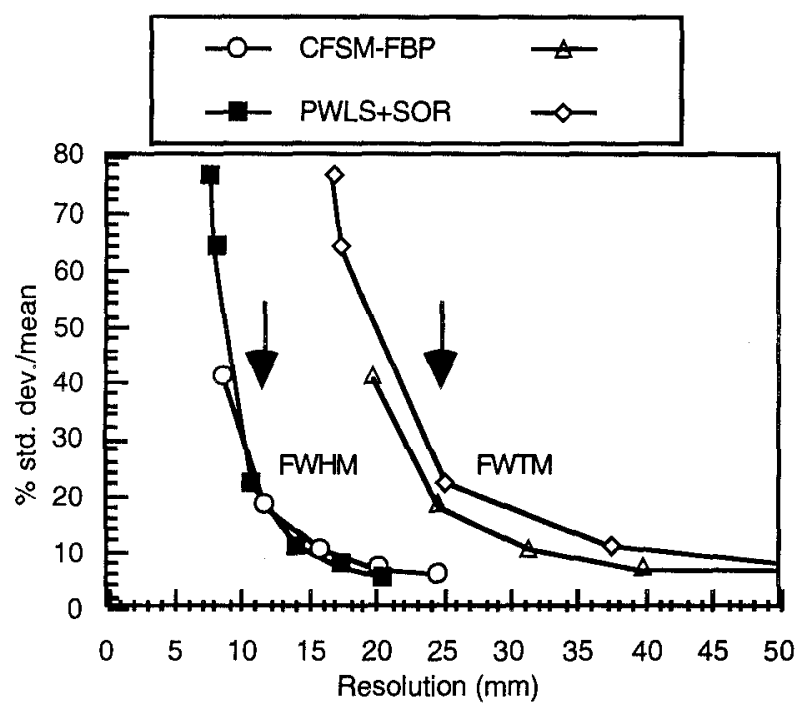

Fig. 7. Background noise vs. resolution as measured by the FWHM and FWTM of a line source in a warm cylinder. The vertical arrows indicate the FWHM and FWTM used for the reconstructions in section $\mathrm{V}$.

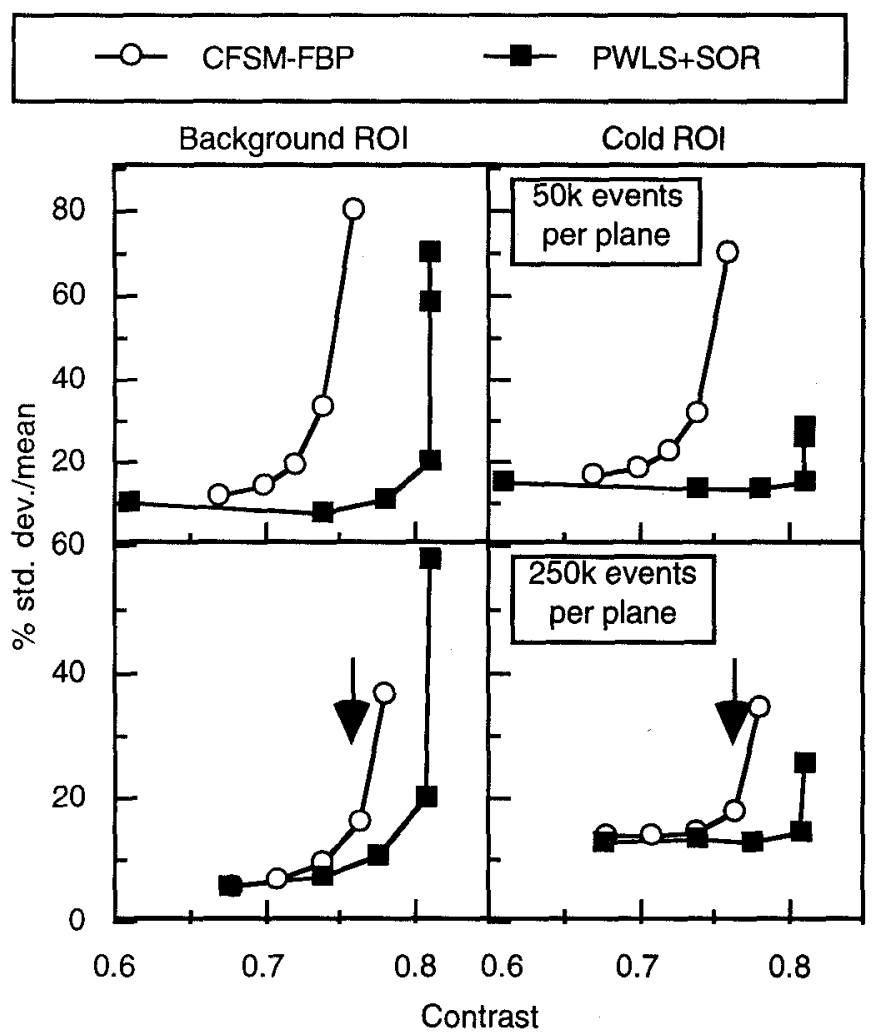

Fig. 8. Contrast vs. noise for two different imaging conditions. Note the change in vertical scale between the two count levels. The vertical arrows indicate the contrast corresponding to the reconstruction parameters used in section $\mathrm{V}$.

Over the range shown in fig. 9, the contrast versus resolution for CFSM-FBP varies linearly for different cut-off frequencies of the Hamming window, while for the PWLS+SOR method there is a non-linear relation between contrast and resolution. The improvement is likely due to both the non-negativity constraint and the incorporation of statistical information.

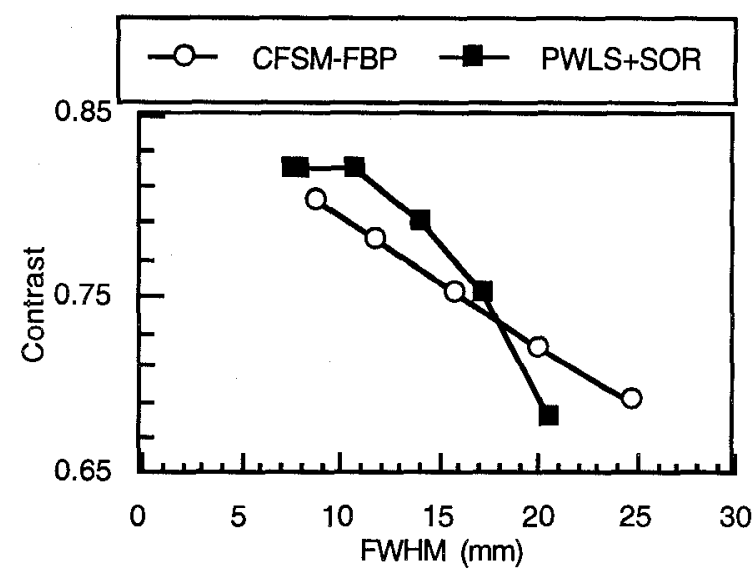

Fig. 9. Contrast vs. resolution (FWHM as calculated by equation 32 as measured from a cylinder with a hot line source, a warm background and a cold insert.

\section{APPLICATION TO WHOLE BODY IMAGING}

\section{A. Phantom Study}

An anthropomorphic torso phantom with hot (10:1 and 3:1 ratios of activity to background concentrations, $1 \mathrm{cc}$ each) and a cold sphere insert $(16 \mathrm{cc})$ was imaged to examine the reduction in noise for the same reconstruction parameters indicated by the arrows in figs. 7 and 8 , which produced a FWHM resolution of $12 \mathrm{~mm}$ for the line source in a warm background as described above. Attenuation correction was performed using a singles transmission source [9].

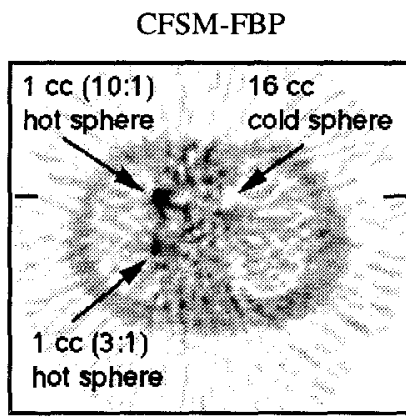

Transverse

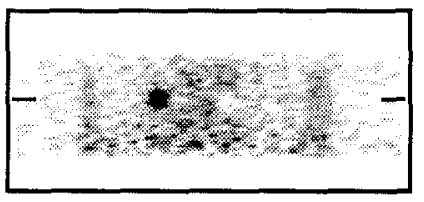

Frontal
PWLS+SOR

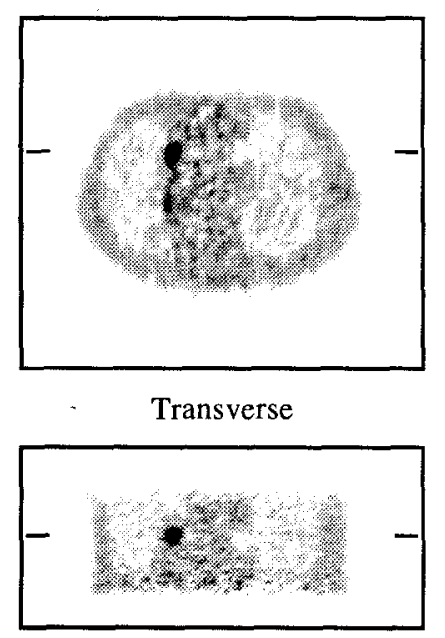

Frontal
Fig. 10. Sections of reconstructed 3D images of an anthropomorphic phantom which has hot and cold sphere inserts. Imaging and reconstruction parameters are similar to those indicated by the arrows in figs. 7 and 8 .

Fig. 10 shows transverse and frontal sections of 3D images reconstructed using both methods. Imaging and reconstruction parameters were similar to those of the whole-body patient study described next to give a basis for comparing the patient images. The reconstruction parameters are typical for wholebody oncology imaging, with the exception of using no axial 
smoothing to allow for a more direct comparison of the two algorithms.

Measured contrast and standard deviation values are given in table 1. Contrast was measured from ROIs of the same diameter as the spherical inserts placed over inserts (determined from a high statistics scan), and calculated as the ratio lbackground - coldl/background. Pixel standard deviation was averaged from large ROIs placed in each of the 'lung' regions of the phantom.

Table 1. Contrast of spheres and standard deviation of lung regions expressed as a percentage of the mean of the warm background.

\begin{tabular}{lcccc}
\hline \hline & \multicolumn{3}{c}{ Contrast } & Std. dev. \\
& $\begin{array}{c}1 \mathrm{cc} \\
\text { hot } \\
(10: 1)\end{array}$ & $\begin{array}{c}1 \mathrm{cc} \\
\text { hot } \\
(3: 1)\end{array}$ & $\begin{array}{c}16 \mathrm{cc} \\
\text { cold }\end{array}$ & \\
\hline Ideal contrast & 9 & 2 & 1.0 & - \\
CFSM-FBP & 6.4 & 1.6 & 0.76 & $\sim 31 \%$ \\
PWLS+SOR & 7.2 & 1.8 & 0.80 & $\sim 20 \%$ \\
\hline \hline
\end{tabular}

Table 1 shows higher contrast ratios for the hot and cold spheres and a lower pixel standard deviation in the lung regions of the phantom.

\section{B. Patient Study}

A whole body study for breast cancer recurrence was performed with typical imaging parameters (approximately $200 \mathrm{k}$ counts per plane). The CFSM-FBP and PWLS+SOR images were reconstructed using the parameters described above. In addition, the same amount of axial Gaussian smoothing (12 mm FWHM) was applied to both of the reconstructed images. The $3 \mathrm{D}$ reconstructed images were 128 voxels on a side, with $2 \mathrm{~mm}$ voxels. Sections through the reconstructed images are shown in fig. 11 and 12 .

While it is not possible to make any conclusions about improved tumor detectability, figs. 11 and 12 show a reduction in statistical noise for PWLS+SOR compared to CFSM-FBP at the resolution shown above (FWHM=12 $\mathrm{mm}$ ).

\section{DISCUSSION}

The relative reconstruction times of the algorithms given in table 2 show that the ratio in reconstruction times between CFSM-FBP and PWLS+SOR is 1:6.5. For the 128-plane whole-body image in fig. 12 the reconstruction time for 20 iterations of SOR is 13 minutes on a DEC Alpha $6005 / 333$ workstation. We observed that the results of the PWLS+SOR method did not change significantly after 10 iterations of the SOR algorithm, which would reduce the computation time to only 6.5 minutes.

Table 2. Relative reconstruction times

\begin{tabular}{ll}
\hline Algorithm & Relative reconstruction time \\
\hline FBP & 1 (by definition) \\
CFSM-FBP & 2.0 \\
PWLS+SOR & 13.0 (for 20 iterations of SOR) \\
\hline \hline
\end{tabular}

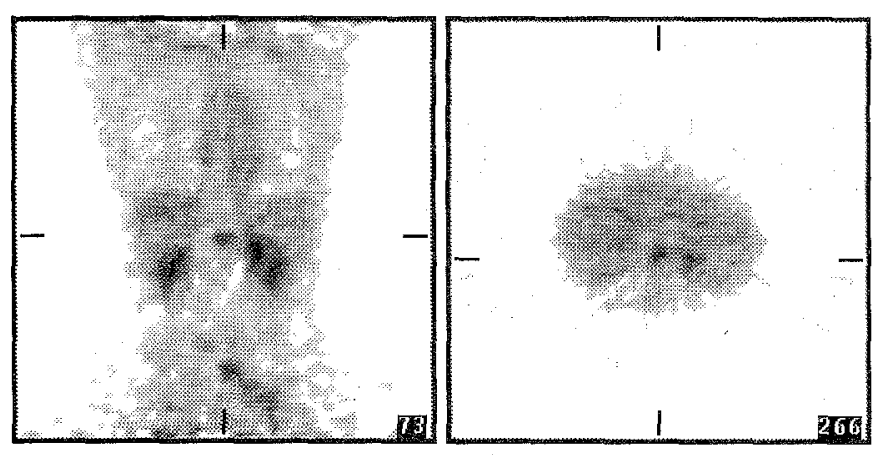

Fig. 11. Frontal and transverse views of a CFSM-FBP reconstruction of a whole body oncology study. Cross hairs are centered on a metastasis from a primary breast tumor.

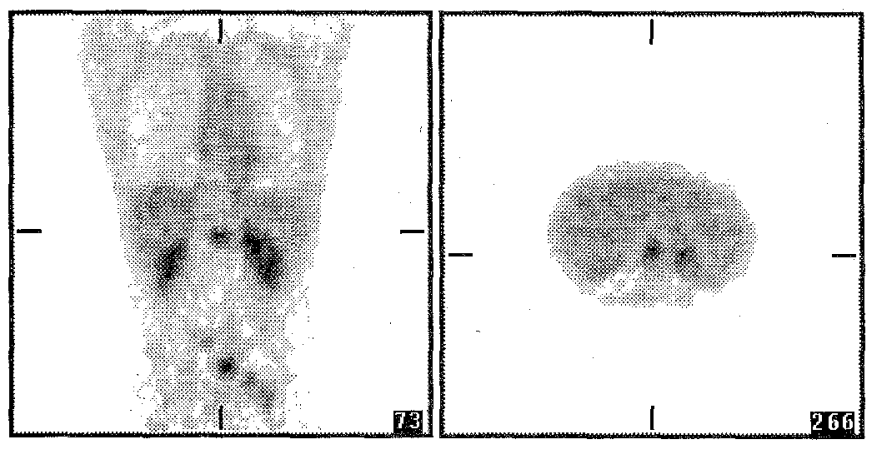

Fig. 12. PWLS+SOR images of the same whole body oncology study shown in fig. 11.

Images reconstructed with PWLS+SOR have favorable noise/contrast tradeoffs compared to those reconstructed with CFSM-FBP due to (i) the non-negativity constraint and (ii) reduced propagation of statistical noise.

While noise calculated as the standard deviation of the background or cold ROIs does not represent the true pixel variance, it can to some extent be considered indicative of trends in image noise. The results obtained here are consistent with a simulation study (for a scanner without detector gaps) where true pixel variance was calculated from multiple realizations and showed that PWLS+SOR had improved biasnoise trade-offs in comparison to FBP [3].

The incorporation of statistical information into the PWLS+SOR method could be done by other statistical methods. Given the imperfect corrections that always occur in practice, however, a statistical model that is insensitive to departures from the assumed shape of the data distribution is probably preferable.

\section{ACKNOWLEDGMENTS}

We thank Gene Gualtieri from UGM Medical Systems for his assistance with the PENN-PET reconstruction software, and Robin Smith, Sean Riggin, and Emannuel Angel from the University of Pennsylvania for their assistance with data acquisition and analysis.

\section{REFERENCES}

[1] Shepp LA and Vardi Y. Maximum likelihood reconstruction for emission tomography. IEEE Trans Med Imag 2:113-119, 1982 
[2] Mumcuoglu EU, Leahy R, and Cherry SR. Bayesian reconstruction of PET images: Methodology and performance analysis. Phys Med Biol 41:1777-1807, 1996.

[3] Fessler JA. Penalized weighted least-squares image reconstruction for positron emission tomography. IEEE Trans Med Imag 13(2):290-300, 1994

[4] Fessler JA and Rogers WL. Spatial resolution properties of penalized-likelihood image reconstruction methods: Space-invariant tomographs. IEEE Trans Imag Proc 5:1346-1358, 1996

[5] Karp JS, Muehllehner G, Mankoff DA et al. Continuousslice PENN-PET: A positron tomograph with volumeimaging capability. J Nucl Med 31:617-627, 1990

[6] Daube-Witherspoon ME and Muehllehner G. Treatment of axial data in three-dimensional PET. $J$ Nucl Med 28:1717-1724, 1987

[7] Karp JS Muehllehner G, and Lewitt RM. Constrained Fourier space method for compensation of missing data in emission computed tomography. IEEE Trans Med Imag $7: 21-25,1988$

[8] O'Sullivan F, Pawitan $Y$, and Haynor D. Reducing negativity artifacts in emission tomography: Postprocessing filtered backprojection methods. IEEE Trans Med Imag 12:653-663, 1993

[9] Karp JS, Muehllehner G, Qu H, and Yan XH. Singles transmission in volume-imaging PET with a $137 \mathrm{Cs}$ source. Phys Med Biol 40:924-944, 1995 